\title{
The Review of English Learning Strategy for Chinese Junior High School Students \\ Shuyan Xu
}

School of Language and Cultural Communication of Shangluo University, Shangluo, Shaanxi 726000

\begin{abstract}
Based on the development of research of English learning strategy, the review are mainly made from three aspects on English learning strategy for Chinese junior high school students, that is theoretical study review, empirical study review and practical study review.
\end{abstract}

Keywords: English learning strategy; Review; Junior high school students

\section{中国初中生英语学习策略研究述评}

\author{
徐淑燕
}

（商洛学院语言文化传播学院，陕西商洛 726000)

摘要: 采用文献分析法, 在回顾研究发展过程基础上, 从理论研究、实证研究和实践探索三个方面对国内初中生英语学 习策略进行了重点述评。

关键词: 英语学习策略; 述评; 初中生

\section{引言}

国外英语学习策略的研究最早开始于 20 世纪 70 年代, 它是在人们意识到教育研究的重点不应只放在 教学方法的改革还应该注重如何提高学生英语水平的研究的背景下开始的。Rubin (1975) 在权威杂志 TESOL Quarterly 第 9 期上发表了颇具影响的研究论文 “ What the Good Language Learner Teach Us” 标志着 西方英语学习策略研究的开始。1984 年黄晓华在香港中文大学完成的以 “ An investigation of learning strategies in oral communication that Chinese EFL learners in China employ ” 为题的硕士论文 标志着中国英语学习策略研究的开端, 随后国内外不少研究者如：Malley，Oxford, Wenden, Cohen 以及 我国的文秋芳、王立非等学者进行了大量的理论和实证研究, 从不同视角研究了英语学习策略。我国对中 学生英语学习策略的研究始于 1997 年章兼中和俞红珍对初中生的单词记忆及对话研究, 随后, 张文鹏等 对中学生的策略使用做过相应的研究 [1], 特别是 2001 年我国新《英语课程标准》颁布以来有越来越多的 人开始从事初中生英语学习策略的研究。目前对初中生英语学习策略的研究已处于全面启动阶段, 在这个 阶段, 研究队伍迅速成长, 研究方法逐步多样, 研究范围明显拓宽, 研究成果不断涌现。据统计, 近十年 来关于初中生英语学习策略研究的文章约有 54 篇. 其中理论研究有 3 篇, 占总数的 $5.6 \%$, 实证研究有 26 篇, 占总数的 $48.1 \%$, 还有 25 篇是关于英语学习策略培养的文章, 占总数的 $46.2 \%$ 。

\section{1 理论研究述评}

\section{1 英语学习策略的定义}

通过已有英语学习策略的文献进行研究发现，对英语学习策略的定义至今没有达成一致意见. 被广泛 接受和采用的主要有以下几种 (见表 1): 
表 1 学习策略的定义归类

\begin{tabular}{|l|l|}
\hline \multicolumn{1}{|c|}{ 研究者 } & 定义 \\
\hline Stern (1983) & 策略指语言学习者所采用方法的一般趋势或总体特点; \\
\hline Chamot (1987) & $\begin{array}{c}\text { 学习策略是学生采取的技巧, 方法或刻意的行动, 其目的是便于学习和 } \\
\text { 回忆语言的形式和内容; }\end{array}$ \\
\hline Rubin (1987) & $\begin{array}{c}\text { 学习策略是有助于学习者自我构建的语言系统发展并直接影响学习的 } \\
\text { 策略; }\end{array}$ \\
\hline 0’ Mal1ey\&Chamot (19 & 学习策略是个体学习者用来理解, 学习或者记住新信息的想法或行为. \\
\hline 90) & \\
\hline 0xford (1990) & 学习策略是学习者从事的一些特定的活动, 目的是使学习更加容易, 更 \\
& 加快捷, 更加有乐趣, 更加自主, 更加能适应新的情况. \\
\hline
\end{tabular}

从表 1 不难看出, 英语学习策略定义存在五大分歧: (1) 策略究竟足外显行为, 还是内隐活动, 还是两 者兼而有之; (2) 策略是有意识的, 还是潜意识的; (3) 策略是指方法, 还是具体的技能; (4) 策略对目标 语学习的影响是直接的, 还是间接的, 还是两者兼而有之; (5) 策略使用的动机是什么。E11is 在以上定义 基础上, 将英语学习策略定义为学习者用以直接或间接提高英语学习效率的内隐或外显方法和技巧, 目前 这一定义已被广大学者接受和认可。

\section{2 英语学习策略的分类}

学习策略的分类和它的定义一样, 由于视角不同, 研究者在英语学习策略的分类问题上也是见仁见智, 争论不休.主要的分类有以下几种 (见表 2):

表 2:学习策略的分类

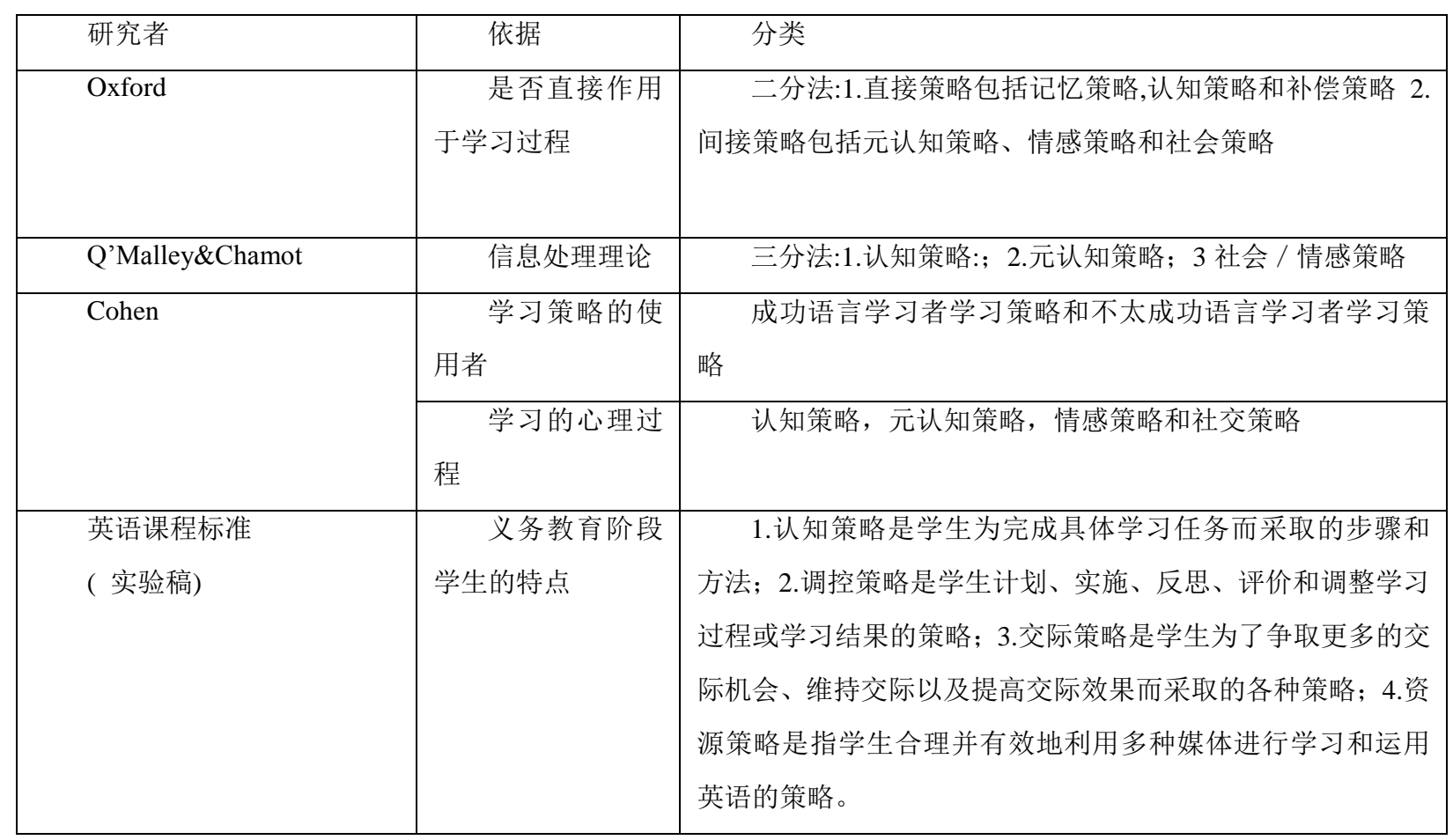

以上分类各有千秋, 但它们有一个共同特点, 即都提出了认知策略, 元认知策略和情感策略. 英语课程 
标准中根据义务教育阶段学生的特点把英语学习策略分为认知策略, 调控策略, 交际策略和资源策略; 这 种分类方法既考虑了策略的使用目的, 又考虑到策略使用的心理和认识过程, 符合初中生英语学习的实际, 因此本文采用的是英语课程标准（实验稿）中对英语学习策略的分类。

\section{3 影响英语学习策略使用的因素}

影响初中生英语学习策略使用的因素很多, 归纳起来主要有: 学习者的因素 (如观念, 年龄, 性别, 动机, 学习时间长短, 学习经历) 和环境因素 (语言环境, 任务, 文化背景等)。现从观念、动机、性别、任务等方面 来分析和归纳了中国学者对初中生英语学习策略使用因素的研究。

1. 观念。观念与外语学习策略使用密切相关已得到大量实证研究的支持。文秋芳等发现观念与策略的 关系有显著相关，但相关程度不等 [2]。其中相关程度最高的是依赖母语观念与依赖母语策略，最低的是 功能操练观念与功能操练策略。跟踪调查的数据表明, 观念与策略的关系有逐年增强的趋势。

2. 动机。杨建新等 [3] 显示动机强的学习者使用英语学习策略的频率高于动机弱的学习者, 并且学习 动机强的学习者更善于使用多种学习策略进行英语学习。

3. 性别。性别也是影响初中生英语学习策略使用的一个重要因素。不同性别的学生在英语学习策略 的选择和使用上存在差异。有发现，女生比男生更常使用记忆策略、元认知策略、情感策略和社交策略; 另有研究显示，男生多使用翻译策略，女生多使用监控策略。苏俊等发现男生比女生更常使用交际策略， 而女生比男生更常使用认知策略，调控策略和资源策略。

4. 任务。策略使用取决于任务类型。策略适合具体的学习任务, 策略使用才有效力。研究表明, 学 习者在进行词汇学习和口语练习时使用策略的数量和频率大于做听力理解和口头报告时使用的策略。 Chamot 等人 (1987) 也发现, 在完成词汇任务时, 学习者倾向于使用认知策略、元认知策略中的自我监控和 自我评估策略; 在完成听力任务时，他们更多使用认知策略中的记笔记和归纳策略、元认知策略中的选择 注意、自我监控和问题确认策略。

\section{4 英语学习策略理论研究特点及展望}

英语学习策略理论研究有如下特点: 第一, 20 世纪 80 - 90 年代是外语学习策略研究的鼎盛时期, 硕 果累累。进入 21 世纪, 策略研究有降温的趋势, 重复性研究较多, 拓展性研究较少。第二, 研究范围比 较狭窄。研究主要集中在策略的定义和分类、策略使用与水平或成绩的关系、策略使用对部分语言技能 (听、 说、读) 的作用以及影响策略使用的因素等几个方面, 而学习策略、策略训练、策略使用对写作技能的作 用以及语音和语法学习策略的研究却不多见。因此, 在今后的研究过程中应该加以创新, 增加拓展性研究, 解决当前英语学习策略理论研究阶段存在的争议。

\section{2 实证研究述评}

\section{1 近十年初中生英语学习策略实证研究现状}

对近十年来研究初中生英语学习策略的实证文章进行统计发现, 发表在国内各类重要期刊的初中生英 语学习策略实证研究的论文共有 23 篇。其中, 有 21 篇研究是关于初中生英语学习策略的使用现状, 占 $91 \%$; 9 篇研究的是农村中学生的英语学习策略的使用现状, 8 篇研究的是城市初中生的英语学习策略的使用现 状, 2 篇对城乡初中生英语学习策略的使用现状作了对比研究. 在这里根据研究内容, 研究对象, 研究方法对 其进行分类(见表 3 )。 
表 3 近十年初中生英语学习策略实证研究归类

\begin{tabular}{|c|c|c|c|c|c|c|c|c|c|c|}
\hline \multirow{3}{*}{ 研究内容 } & \multicolumn{5}{|c|}{ 研究对象 } & \multicolumn{4}{|c|}{ 研究方法 } & \\
\hline & \multirow{2}{*}{$\begin{array}{l}7 \\
\text { 年 } \\
\text { 级 }\end{array}$} & \multirow{2}{*}{$\begin{array}{l}8 \\
\text { 年 } \\
\text { 级 }\end{array}$} & \multirow{2}{*}{$\begin{array}{l}9 \\
\text { 年 } \\
\text { 级 }\end{array}$} & \multirow{2}{*}{$\begin{array}{l}\text { 初 } \\
\text { 中 }\end{array}$} & \multirow{2}{*}{$\begin{array}{l}\text { 混 } \\
\text { 合 }\end{array}$} & \multicolumn{3}{|c|}{ 问卷调查 } & \multirow{2}{*}{$\begin{array}{c}\text { 混合 (问卷 } \\
\text { 调查, 测试, } \\
\text { 访谈) }\end{array}$} & 论 \\
\hline & & & & & & $\begin{array}{c}\text { Oxford 问 } \\
\text { 卷调查 }\end{array}$ & $\begin{array}{l}\langle\text { 新课标〉〉 } \\
\text { 问卷调查 }\end{array}$ & $\begin{array}{l}\text { 自制问 } \\
\text { 卷调查 }\end{array}$ & & 遙 \\
\hline $\begin{array}{c}\text { 初中生英语学 } \\
\text { 习策略使用现 } \\
\text { 状分析 }\end{array}$ & & 3 & 4 & 10 & 4 & 1 & 10 & 8 & 2 & 21 \\
\hline $\begin{array}{l}\text { 初中生英语学 } \\
\text { 习策略与成绩 } \\
\text { 的相关性研究 }\end{array}$ & 1 & & & & 1 & & & & & 1 \\
\hline $\begin{array}{c}\text { 成败归因对初 } \\
\text { 中生英语学习 } \\
\text { 策略选择的影 } \\
\text { 响 }\end{array}$ & 1 & & & & 1 & & & 1 & & 1 \\
\hline 合计 & 2 & 3 & 4 & 10 & 4 & 1 & 10 & 9 & 3 & 23 \\
\hline
\end{tabular}

\section{2 近十年初中生英语学习策略实证研究分析}

（1）从作者来看, 近十年来有 31 人对初中生英语学习策略作出了一定的贡献, 其中苏俊, 李小芬, 熊广 星三人 3 次在重要期刊上发表关于研究初中生英语学习策略的文章。另外, 在这 31 人中除了 4 人是硕士研 究生外其余 基本上是中学教师。由此可见, 我国中学教师越来越重视培养学生的英语学习策略运用能力。

(2) 从研究对象方面分析。从研究对象来看, 2 篇是关于初一学生, 3 篇是初二学生, 4 篇是初三学生, 4 篇是中学生 (初一至高三学生), 其余 10 篇都是研究从初一到初三 整个初中阶段. 其中有 9 篇是研究农村初 中生, 8 篇是研究城市初中生, 2 篇对城乡初中生做了对比研究。由此可见, 当前对初中生英语学习策略的研 究较为全面, 涉及到中学阶段的各个年级和不同群体。

（3）研究方法方面分析。就研究方法而言, 大都采取的是问卷调查的方式, 其中有 10 篇采用的是新《英 语课程标准》中 7 至 9 年级学生英语学习策略评价表, 1 篇采取的是 $0 x f o r d$ 的〈〈语言学习测量表〉〉, 9 篇是 自制调查问卷。问卷调查占了策略研究的主体。从本质说, 问卷调查只能提供描述性数据, 例如研究不同 年级、不同性别在英语学习观念和行为上表现出的趋势, 以及这些趋势与成绩的相关性时我们还需要更多 的实验研究、个案研究或者混合法研究，从深层次上考察学习策略对英语学习的影响。

(4) 研究内容方面分析。从研究内容方面来看, 有 21 篇研究的是初中生英语学习策略的使用现状及不 同年级, 不同性别英语学习策略使用情况的差异; 王文慧 (2008) 研究了初中生英语学习策略与英语成绩的 相关性 [4]; 黄水美 (2006) 研究了农村初中生元认知英语学习策略 [5]。可见，本文所涉及的 23 篇论文都 是从宏观角度考察初中生英语学习策略的使用状况及其使用模式, 性别与学习策略使用之间的差异, 学习 策略之间的相关性, 以及学习策略对英语成绩的作用。但是, 对某一类知识或者技能策略的研究, 如听力 
策略、阅读策略、词汇策略、口语策略和写作策略的研究却是空白。

\section{3 近十年初中生英语学习策略实证研究结果分析}

这里本文将根据研究视角的不同对 23 项实证研究的结果分别评述。

（1）初中生英语学习策略使用现状。研究初中生英语学习策略使用现状的文章有 21 篇, 通过对这 21 篇文章的研究结果进行分析发现它们有以下几点相同之处：(1) 初中生使用学习策略的整体水平一般; (2) 初中学生使用学习策略的多样性不够大, 使用策略的频率不够高; (3) 女生比男生更频繁的使用多种策略; （4）英语学习策略的使用情况与英语学习成绩呈正相关; (5) 不同地区, 不同年级, 不同性别的学生的学习策 略使用情况差异较大; (6) 城乡初中生英语学习策略的使用水平都较差。但是这些文章在初中生英语学 习策略使用的基本模式方面仍没有一致结果。有研究显示, 初中生使用最频繁的是元认知策略, 另有研究 显示是情感策略，而赵淑娜（2011）认为是资源策略使用最频繁 [6]。

（2）初中生英语学习策略与成绩的相关性研究。近十年来研究初中生英语学习策略与成绩的相关性的 文章只有一篇。王文慧 ( 2008) 研究表明学习策略是影响英语成绩的重要因素。同时还发现英语成绩与学 习策略之间显著相关, 认知策略目前对中学生英语成绩影响较大。因此, 教师应在教学中加强对英语学习 策略的指导和训练, 以提高学生使用这些策略的水平进而提高学生的英语学习成绩。

（3）成败归因对初中生英语学习策略选择的影响。自 90 年代起，对归因和学习策略的研究引起广泛 的关注并被视为中国教育心理学和学习心理学的一大收获。但是, 对于外语学习情景下学习策略的使用与 归因的关系方面的研究甚少。近十年来研究这一问题的文章只有一篇, 研究发现, 大多数学生都能够正确 归因, 不同成绩的初中学生都选择内部因素来解释自己的成功或失败; 归因不仅能通过学习策略影响学习 成绩, 而且能直接影响学习成绩。根据以上的调查结果, 本研究对初中英语教学提出几点建议: 促进学生 英语学习中社会策略的使用; 进一步提高学生元认知意识; 了解学生的归因倾向, 引导学生正确归因。

\section{4 问题与不足}

近 10 年来关于初中生英语学习策略研究领域已取得了一些成就, 但存在的问题还不少, 主要的问题 有: (1) 对初中生英语学习策略运用现状的研究已经趋于饱和, 但对英语学习策略与成绩的相关性研究以及 英语学习策略选择的影响因素的研究却很少。(2) 对某一类知识或者技能策略的研究, 如听力策略、阅读 策略、词汇策略、口语策略和写作策略的研究几乎是空白; (3) 使用的研究方法种类严重不均衡。问卷调 查远远多于实验研究、个案研究和混合研究。

\section{3 实践探索述评}

学习策略的使用对信息的存储、修正及使用，对提高学习者自主能力、独立能力和自我指导能力有极 大作用, 它能关系到英语学习者能否有有效学习英语及能否获得终身学习能力的一个重要因素。素质教育 强调教师应加强学生获取运用知识的能力, 分析解决问题的能力, 由此可见, 英语学习策略培训和培养学 生独立、自主、有效运用学习策略的能力, 不仅十分必要, 而且具有现实指导意义。本文对近十年关于初 中生英语学习策略培养的 25 篇文章进行了研究, 研究发现在初中生英语学习策略培训的研究中主要是从学 生和教师两个方面对英语学习策略培训的途径和方法作了介绍 [7]。王飞等 [8]在硕士论文中对当前初中生 的英语学习策略培训现状进行了调查研究并针对现状提出了进行英语学习策略培训的方法以及培训中应 该注意的问题。 
通过对中学生英语学习策略培训的研究发现当前中学生英语学习策略培训存在着一些不足: (1) 当前 中学教师对学生英语学习策略的培训不够重视; (2) 当前初中生英语学习策略培训形式单一, 主要是在课 堂教学过程中进行简单的策略培训, 由于缺乏系统性所以培训成效不高. 因此, 教育工作者应该加强对学生 进行英语学习策略的培训;（3）教育者忽视了对初中生进行社会情感因素的培训。针对我国中学生英语学 习策略的使用及培训现状, 英语学习策略的培训应该注意以下几点: (1) 首先应该了解学生的性格特点, 学 习特点, 并根据他们的情况制定学习策略培训的内容; (2) 通过不同的内容制定不同的培训方法. 例如在进 行听力策略培训的过程中应该将培训融入到听力教学之中; 在进行交际策略培训的过程中就应该进行长时 间的培训和练习相结合; 在进行阅读策略的培训时首先应该进行专门的方法的培训, 随后要针对这一策略 进行反复的训练以便更好的掌握这一策略; (3) 对学生进行必要的情感策略和社会策略培养有助于调动学 生学习英语的积极性, 激发他们学习策略的强烈欲望进而对提高学生英语学习水平起到重要的作用。另外, 对学生进行情感策略的培养能够提高学生的综合能力, 有助于个人更好的发展;（4）坚持学习策略培训与 语言技能培养并重的原则，注重策略教学与常规教学的有机结合，切忌顾此失彼或厚此薄彼。

\section{4 结语}

学习策略对学生有效的学习英语起着至关重要的作用。良好的英语学习策略不仅有助于学生把握学习 方向, 提高学习效率, 而且有助于他们形成自主性学习的能力, 为终身学习奠定基础。初中阶段是英语学 习的基础阶段同时也是一个非常重要的阶段, 初中生掌握一定的英语学习策略对其有效地进行英语学习是 非常重要的。为了了解当前我国初中生英语学习策略研究现状和使用现状, 学习一些英语学习策略培训方 法, 本文从理论、实证和实践探索三方面综述了近十年来我国初中生英语学习策略研究, 并提出了自己的 看法, 希望会对中学教师的英语教学以及自己以后的教学工作有所帮助. 总体看来, 初中生英语学习策略研 究有如下特点:第一, 自 2003 年以来初中生英语学习策略研究有增温趋势, 但重复性研究较多. 拓展性研究 较少; 第二, 研究范围较窄, 内容较单一; 第三, 研究深度和广度不够, 研究多热衰于宏观研究, 较少关注具 体策略的研究; 第四, 研究方法不够完善. 实证研究较多, 理论研究较少; 使用调查问卷方式的研究较为普遍, 使用综合方式的较少。

近十年来中国学者对初中生英语学习策略研究取得了一定的成果, 但总体来说初中生英语学习策略研 究还处于起步阶段, 可供参考的研究成就还比较贵乏。所以希望更多研究者和中学教师能关注这个领域, 在 英语教学心理学领域 [9] 有更多有影响的研究成果发表, 从而促进中学英语教学质量的提高, 达到提升初中 生英语学习能力和自主学习能力的效果。

\section{5 致谢}

本项目为陕西省教育科学 “十二五”规划 2013 年立项课题 (SGH13402); 商洛学院科研基金项目 (14SKY-FWDF010) 支持项目。

\section{Acknowledgement}

This project is the support project of Shaanxi Provincial Education Science "Twelve-five" Plan in 2013; the Scientific Research Fund Project (14SKY-FWDF010) of Shangluo College.

\section{参考文献:}

[1] 张文鹏. 外语学习动力与策略运用之关系 $[\mathrm{J}]$. 外语与外语教学, 1998, (3). 
[2] 文秋芳. 英语学习者动机、观念、策略的变化规律与特点 $[J]$. 外语教学与研究, 2001, (2).

[3] 杨建新. 任务驱动下的大学英语学习自主性培养 $[J]$. 河西学院学报, 2013, (3).

[4] 王文惠. 从教与学两方面衔接好小学和初中英语 $[J]$. 现代中小学教育, 2007, (1).

[5] 黄水美. 农村初中生元认知英语学习策略调查分析 $[J]$. 山东师范大学外国语学院学报, 2006, (2).

[6] 赵淑娜. 初中生使用英语学习策略的调查研究 [J]. 教育实践与研究 (B), 2011, (9).

[7] 王国荣. 初中生英语学习策略研究与培养[D]. 山东师范大学：山东师范大学, 2008.

[8] 王飞. 初中生英语学习策略培训的研究 [D]. 东北师范大学：东北师范大学, 2007.

[9] 徐淑燕, 杨宪华. 我国英语教学心理学研究文献计量分析 $[J]$. 商洛学院学报, 2014, (5).

\section{References:}

[1] Zhang Wenpeng. The Relationship between Foreign Language Learning Motivation and Strategy Use [J].Foreign Languages and Their Teaching, 1998, (3).

[2] Wen Qiufang. Changing Rules and Characteristics English Learners' Motivation, Beliefs and Strategies [J].Foreign Language Teaching and Research, 2001, (2).

[3] Yang Jianxin. Task - Driven Cultivation of Learner Autonomy in College English [J].Journal of Hexi University, 2013, (3).

[4] Wang Wen-Hui. Well Matched in Both Teaching and Learning of Primary and Junior Middle School English [J].Modern Primary and Secondary Education,2007,(1).

[5] Huang Shuimei. Investigation and Analysis of Rural Junior Middle School Students' Metacognitive Learning Strategies [J].Journal of School of Foreign Languages of Shandong Normal University, 2006, (2).

[6] Zhao Shuna. An investigation on English Learning Strategies of Junior Middle School Students' Use [J].Education Practice and Research(B),2011,(9).

[7] Wang Guorong. Research and Cultivation of Junior Middle School Students' English Learning Strategies [D].Shandong Normal University: Shandong Normal University, 2008.

[8] Wang Fei. Research on Junior Middle School Students' English Learning Strategies [D].Northeast Normal University: Northeast Normal University, 2007.

[9] Xu Shuyan, Yang Xianhua. Bibliometric Analysis of English Teaching Psychology Research in China [J].Journal of Shangluo University, 2014,(5). 